\title{
APLICACIONES EMERGENTES DE INTELIGENCIA COMPETITIVA EN LAS UNIVERSIDADES
}

\author{
Montserrat Garcia-Alsina, Eva Ortoll y Alexandre López-Borrull
}
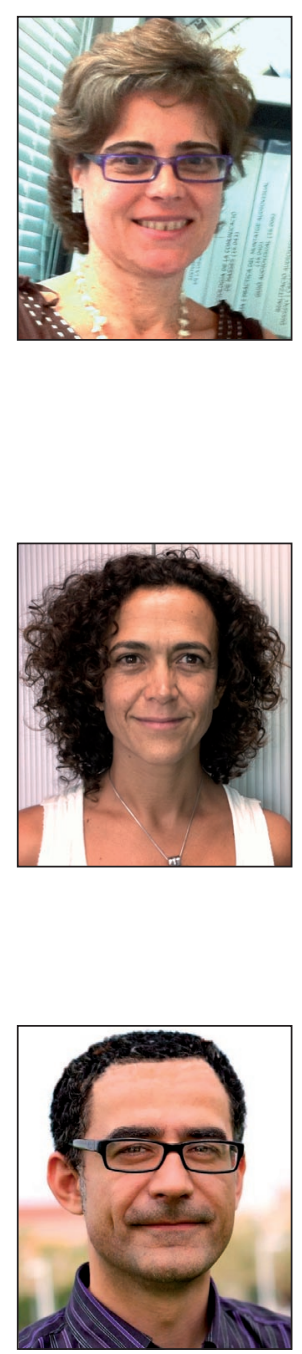

Montserrat Garcia-Alsina es profesora de los Estudios de Ciencias de la Información y de la Comunicación, en el programa de Documentación de la Universitat Oberta de Catalunya (UOC) e investigadora del grupo $\mathrm{KIMO}$ (Knowledge and Information Management in Organisations, grupo consolidado de la Generalitat de Catalunya). Es licenciada en documentación por la UOC, y su materia de investigación es la gestión documental, la gestión del conocimiento, la gestión de la información, y en especial la inteligencia competitiva tanto en las administraciones públicas como en el sector empresarial. Su tesis doctoral versa sobre la contribución de la inteligencia competitiva en el proceso de adaptación de las universidades al Espacio Europeo de Educación Superior.

Universitat Oberta de Catalunya (UOC) Estudios de Ciencias de la Información y de la Comunicación Rambla del Poble Nou, 156. 08018 Barcelona http://www.uoc.edu/estudios/grados/infodocu mgarciaals@uoc.edu

Eva Ortoll es profesora agregada en los Estudios de Información y Documentación de la Universitat Oberta de Catalunya (UOC), e investigadora del grupo KIMO (Knowledge and Information Management in Organisations) de la misma universidad. Doctora en sistemas de información y documentación por la Universidad de Zaragoza, licenciada en documentación por la Universidad Carlos III de Madrid y diplomada en biblioteconomía por la Universitat de Barcelona. Como investigadora ha trabajado en temas de inteligencia competitiva, gestión estratégica de la información, comportamiento de usuarios y gestión del conocimiento. Recientemente ha sido investigador visitante en la Loughborough University (Reino Unido).

Universitat Oberta de Catalunya (UOC) Estudios de Ciencias de la Información y de la Comunicación Rambla del Poble Nou, 156. 08018 Barcelona http://www.uoc.edu/estudios/grados/infodocu eortoll@uoc.edu

\begin{abstract}
Alexandre López-Borrull es profesor agregado en los Estudios de Información y Documentación de la Universitat Oberta de Catalunya (UOC). Doctor en química por la Universitat Autònoma de Barcelona y profesor ayudante en la misma universidad en el período 1998-2009. Licenciado en documentación por la UOC. Como investigador ha trabajado en temas de aspectos legales de la información, fuentes de información electrónica en ciencia y tecnología, y ha participado en diversos proyectos de investigación. Forma parte del grupo de investigación KIMO (Knowledge and Information Management in Organisations) de la UOC.
\end{abstract}

Universitat Oberta de Catalunya (UOC) Estudios de Ciencias de la Información y de la Comunicación Rambla del Poble Nou, 156. 08018 Barcelona http://www.uoc.edu/estudios/grados/infodocu alopezbo@uoc.edu

\section{Resumen}

Distintos autores abogan por la importancia de aplicar la inteligencia competitiva a sectores no empresariales (administración pública, universidad, instituciones no gubernamentales, etc.). De hecho en las universidades se aplican cada vez más herramientas y técnicas de gestión propias de la empresa, pues deben rendir cuentas de los recursos que reciben de la sociedad, y ser más eficaces. Por otra parte, reciben presiones para reformar su oferta formativa según las necesidades de la sociedad y las demandas del mercado laboral. Ello se debe tanto a su implicación en el desarrollo de la región donde están ubicadas, como por el conocido proceso de adaptación de las titulaciones al Espacio Europeo de Educación Superior. Se describen y caracterizan las prácticas de IC en las universidades, analizando su contribución al diseño de los grados. La investigación se basa en métodos cualitativos, empleando entrevistas para recoger datos. Los resultados principales del estudio sugieren que el empleo de inteligencia competitiva en las universidades es principalmente reactivo y sin mayor alcance que la simple táctica organizativa.

\section{Palabras clave}

Inteligencia competitiva, Gestión de información estratégica, Vigilancia del entorno, Oferta universitaria, Reforma de la educación superior, Fuentes de información, Espacio europeo de educación superior, EEES. 
Title: Emerging applications of competitive intelligence in the universities

\section{Abstract}

Different authors have pointed out the importance of applying competitive intelligence (Cl) to non-business sectors (public administration, universities, non-governmental organizations, etc.). Besides, universities increasingly apply management tools and techniques coming from the business field. On the other hand, in recent years universities have perceived an environmental pressure as a consequence of a) their implication in regional development where they are located, and b) their need to reform their program offerings according to the needs of society and the requirements of the labour market, as well as a result of the process of adapting their degrees to the European Higher Education Area (Bologna process). This paper describes and characterises $\mathrm{Cl}$ practices in the universities and analyses the $\mathrm{Cl}$ contribution to the design of degree courses. The research is based on qualitative methods, and data were collected through interviews. Results suggest that the use of $\mathrm{Cl}$ in universities is mainly reactive, and addressed only to organisational tactics.

\section{Keywords}

Competitive intelligence, Business intelligence, Environmental scanning, Strategic information management, European higher education area, EHEA process, Human information sources.

Garcia-Alsina, Montserrat; Ortoll, Eva; López-Borrull, Alexandre. "Aplicaciones emergentes de inteligencia competitiva en las universidades". El profesional de la información, 2011, septiembre-octubre, v. 20, n. 5, pp. 503-509.

http://dx.doi.org/10.3145/epi.2011.sep.03

\section{Introducción}

Como es lógico, las universidades perciben los retos provenientes de su entorno, a los cuales deben hacer frente. En primer lugar, el papel de las universidades en el desarrollo regional ha sido considerado a partir de su misión (docencia, investigación y transferencia de conocimiento) y del vínculo universidad, industria y gobierno, un triángulo conocido también por triple-hélice, que pasa por ser el motor de la innovación. Este papel se ha apuntado tanto desde ámbitos gubernamentales, como desde consejos de gobierno de las universidades y desde la Comisión Europea.

En segundo lugar, durante los últimos veinte años las universidades han experimentado un incremento de la presión del entorno, originado por la competencia de otras universidades, la existencia de nuevos modelos de financiación, y la introducción en sus sistemas educativos de elementos de mercado (Larsen; Maassen; Stensaker, 2009).

En tercer lugar, en los últimos 10 años, las universidades han tenido que reformar su oferta formativa acorde al proyecto de configuración del Espacio Europeo de Educación Superior (EEES), siguiendo los acuerdos adoptados en la Declaración de la Sorbona de 1998 y la Declaración de Bolonia de 1999, suscritas por los ministros europeos de educación.

Para hacer frente a estos imperativos se ha apuntado la necesidad de adoptar nuevas herramientas que orienten la estrategia de la universidad para obtener ventaja competitiva, y también sistemas de información que permitan observar el entorno (Cobarsí; Bernardo; Coenders, 2008; Hammond; Harmon; Webster, 2007).

En este escenario algunos autores apuntan el papel de la inteligencia competitiva (IC) como una de las herramientas de gestión provenientes del mundo empresarial, apropiada también para la planificación estratégica de las universidades, y para la adaptación de éstas a los cambios del entorno
(Ortoll et al., 2008; Cronin, 2006). No obstante, desconocemos las peculiaridades de la aplicación de la IC en el sector universitario, por la escasez de estudios empíricos en este ámbito.

El objetivo de la presente investigación es analizar y describir la aplicación de la IC -la función y el ciclo de inteligencia-en las universidades españolas para definir su estrategia en el diseño de las titulaciones para adaptarse al EEES.

Al conocer la contribución de la IC en la esfera de la oferta formativa, dibujamos un modelo que constituye un punto de partida para un estudio futuro de aplicación de IC en otras áreas de gestión de la universidad, como son definición de líneas de investigación, búsqueda de socios para llevar a cabo proyectos, atracción de estudiantes, o localización de empresas interesadas en la transferencia tecnológica.

\section{Las actividades de inteligencia competi- tiva en las universidades son incipientes, y en general han sido sólo reactivas y como táctica a corto plazo}

\section{Metodología}

La investigación es exploratoria y está diseñada a partir de métodos cualitativos, tanto para la selección de la muestra como para la recogida y análisis de datos y la elaboración de resultados.

\subsection{Selección de la muestra}

De todas las universidades existentes en España tomamos como universo del estudio las que en 2009 ya habían participado en el proceso de adaptación de los grados de EEES, independientemente del estadio y del resultado obtenido en la verificación de la titulación. De este universo, constituido 


\begin{tabular}{|c|c|c|}
\hline $\begin{array}{l}\text { Universidades } \\
\text { participantes }\end{array}$ & $\begin{array}{c}\text { Ramas de } \\
\text { conocimiento }\end{array}$ & Grados \\
\hline \multirow{4}{*}{$\begin{array}{l}\text { A Coruña } \\
\text { Antonio de Nebrija } \\
\text { Autònoma de Barcelona }\end{array}$} & $\begin{array}{l}\text { Artes y } \\
\text { humanidades }\end{array}$ & Humanidades \\
\hline & Ciencias & $\begin{array}{l}\text { Física } \\
\text { Matemáticas }\end{array}$ \\
\hline & $\begin{array}{l}\text { Ciencias de la } \\
\text { salud }\end{array}$ & Medicina \\
\hline & \multirow{9}{*}{$\begin{array}{l}\text { Ciencias } \\
\text { sociales y } \\
\text { jurídicas }\end{array}$} & Terapia ocupacional \\
\hline \multirow{5}{*}{$\begin{array}{l}\text { Navarra } \\
\text { Oberta de Catalunya }\end{array}$} & & Sociología \\
\hline & & $\begin{array}{l}\text { Comunicación audio- } \\
\text { visual }\end{array}$ \\
\hline & & Derecho \\
\hline & & $\begin{array}{l}\text { Publicidad y relaciones } \\
\text { públicas }\end{array}$ \\
\hline & & $\begin{array}{l}\text { Información y docu- } \\
\text { mentación }\end{array}$ \\
\hline Pablo de Olavide & & Periodismo \\
\hline Pompeu Fabra & & Economía \\
\hline \multirow[t]{2}{*}{ Pública de Navarra } & & Psicología \\
\hline & \multirow{3}{*}{$\begin{array}{l}\text { Ingeniería y } \\
\text { arquitectura }\end{array}$} & Arquitectura \\
\hline \multirow[t]{2}{*}{$\begin{array}{l}\text { Ramón Llull } \\
\text { Sevilla } \\
\text { Zaragoza }\end{array}$} & & $\begin{array}{l}\text { Ingeniería y gestión de } \\
\text { las TIC } \\
\text { Ingeniería industrial } \\
\text { Ingeniería de telecomu- } \\
\text { nicaciones }\end{array}$ \\
\hline & & $\begin{array}{l}\text { Ingeniería informática y } \\
\text { gestión }\end{array}$ \\
\hline
\end{tabular}

Tabla 1. Universidades y titulaciones elegidas para el estudio

por la práctica totalidad de las existentes, seleccionamos 13 universidades y 18 titulaciones (tabla 1) con el criterio de dar cabida al mayor número posible de casuísticas existentes, tanto por su situación interna como por su entorno. Se procuró que hubiera:

a) diversidad de comunidades autónomas, en cuanto a número de universidades existentes en cada una, volumen demográfico y extensión territorial, para dar cabida a circunstancias como percepción de competencia, presión demográfica o diversidad de oferta formativa;

b)año de fundación para incluir tanto a universidades históricas como a universidades de reciente creación;

c) titularidad pública y privada atendiendo a diferentes modelos de gestión;

d)perfil de la oferta formativa en cuanto a si un centro presentaba una titulación nueva en su universidad, o en el catálogo español, o si la titulación presentada era una adaptación de una ya existente;

e) representatividad de todas las ramas de conocimiento, por la diversidad de demanda en cada una de ellas;

f) premura sentida por cada titulación, según la convocatoria en la que ha participado, que hubiera podido dar lugar a un contexto de urgencia y precipitación.

\subsection{Recogida de datos: entrevistas}

Se hicieron entrevistas abiertas y focalizadas, con guión preestablecido, con el objetivo de explorar las características de las universidades y sus prácticas de IC. En total entrevistamos a 43 personas, pertenecientes tanto al consejo de gobierno de la universidad, como a las facultades o departamentos que habían efectuado la adaptación de un grado.
Las entrevistas, cuya duración osciló entre 45 y 120 minutos, se estructuraron en dos bloques. El primero recogía aspectos organizativos sobre la universidad, la facultad o el departamento, concretamente: sus objetivos estratégicos, su competencia, sistemas de información existentes y la vinculación con el entorno. El segundo incluía las cuestiones relacionadas con el proceso de verificación: a) cómo organizaron la búsqueda de información y su análisis; b) cuáles eran los temas de información clave; y c) qué fuentes de información utilizaron. Finalmente preguntamos sobre la contribución de la IC al proceso global.

Una vez efectuadas transcribimos las entrevistas y las analizamos mediante el software de asistencia al análisis de los datos cualitativos Atlas.ti (http://www.atlasti.com). Para ello, definimos un conjunto de códigos correspondientes a los temas antes apuntados.

\section{Marco de análisis}

Debido a la escasez de investigación sobre inteligencia competitiva en las universidades, diseñamos el marco de análisis a partir de los modelos existentes en la literatura para organizaciones del sector privado e industrial. Concretamente consideramos dos materias: función de inteligencia y ciclo de generación de la inteligencia, para cada una de los cuales se contó con un conjunto de indicadores (figura 1).

\subsection{Función}

Para analizar la inteligencia como función organizativa nos hemos centrado en tres características:

- orientación a nivel organizativo táctico (actividad diaria o a corto plazo) o estratégico (actividad a largo plazo) (Fahey, 2007; Culver, 2006);

- ubicación de la función, concretamente si está en una unidad dedicada sólo a inteligencia, o en otra junto con otra función organizativa. Además hemos tenido en cuenta el punto de vista de la centralización o descentralización de la función, relacionándolo con la orientación táctica o estratégica (Sawka, 2001). La carencia de una unidad formal o la descoordinación entre unidades que efectúan funciones de IC ocasionan duplicidad de datos y de esfuerzos en la recogida, tratamiento y difusión de información, o menor recogida, análisis y uso de productos de inteligencia y servicios (Heppes; Du Toit, 2009; Fahey, 2007);

- frecuencia en observar el entorno, pues eso influye en la orientación táctica o estratégica de la función. Los sistemas de inteligencia con periodicidad de observación irregular son reactivos y están encaminados a resolver problemas específicos tácticos. Aunque presentan cierta proactividad, predomina la orientación reactiva. Por su parte, los sistemas de observación continua son proactivos y están orientados a detectar oportunidades y a diseñar acciones estratégicas (Cartwright; Boughton; Miller, 1995).

A estas tres características añadimos la percepción del entorno y la incertidumbre percibida porque influyen en las actitudes y en la frecuencia de la observación. De acuerdo con la teoría del sense-making de Weick ${ }^{1}$, la manera en que las organizaciones perciben su entorno determina cómo aplican y organizan el proceso de inteligencia competitiva (El Mabrouki, 2007). 


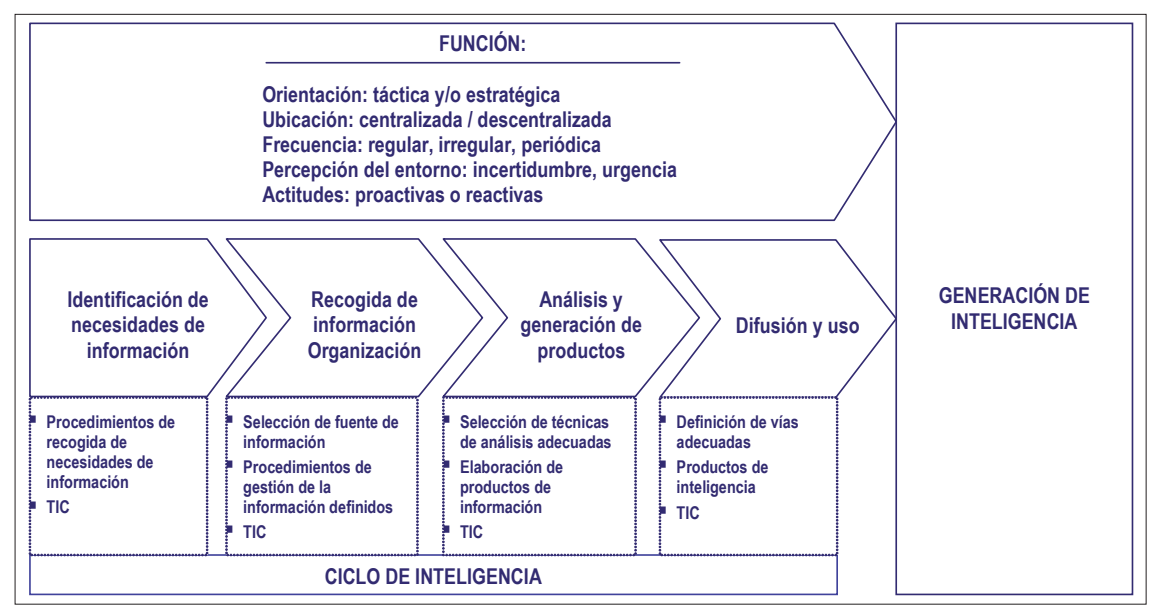

Figura 1. Cadena de inteligencia competitiva en las organizaciones: aspectos de la función y el ciclo analizados en la función de inteligencia (Fleisher; Bensoussan, 2007), al ser el proceso de análisis el que añade valor, y donde la información se transforma en inteligencia para la acción. En la difusión es importante tener unas vías establecidas y elaborar productos de inteligencia en forma de dossiers, informes o análisis de asuntos estratégicos. Estos productos contribuyen a la toma de decisiones y a la planificación estratégica. La eficacia de la difusión depende también de la tecnología y de los sistemas de información implementados (ERP, CRM, datawarehouse), correos electrónicos e intranets (Marin; Poulter, 2004; Choo, 2002).

\subsection{Ciclo de inteligencia}

A pesar de que existen varios modelos para definir el ciclo de IC, en general se acepta que la IC es un proceso iterativo-de aproximaciones sucesivas- que comprende las fases siguientes: 1) Identificación de necesidades de inteligencia; 2) recogida de información y organización, 3) análisis de información y generación de productos de inteligencia para la acción; y 4) difusión de inteligencia y uso de ésta en la planificación estratégica y toma de decisiones (Dishman; Calof, 2008; Ortoll; Garcia, 2008; Bergeron; Hiller, 2002). Partimos de este marco como base para analizar los datos obtenidos en nuestra investigación.

Cada fase tiene características clave que influyen en la eficacia del sistema de inteligencia. En la primera es importante la existencia de procedimientos para inventariar y actualizar las necesidades tácticas y estratégicas. Para recoger y organizar información es importante identificar las fuentes adecuadas, y disponer de sistemas para su organización y almacenamiento.

Las fuentes de información pueden estar disponibles en línea (redes sociales, bases de datos tanto de pago como gratuitas...), o ser fuentes humanas (humint o human intelligence). Éstas juegan un destacado papel, tanto las internas constituidas por los empleados de la organización, como las externas, constituidas por su capital social (Ortoll et al. 2010; Fleisher, 2008; Michaeli, 2006). En la fase de análisis es fundamental seleccionar las técnicas adecuadas a los objetivos señalados

\section{Resultados}

En este apartado describimos las prácticas de inteligencia que se realizaban en las universidades españolas. En concreto describimos los siguientes indicadores de la función y del ciclo de inteligencia: 1) organización de la función de inteligencia de acuerdo con las necesidades de información manifestadas; 2) aspectos de la recogida de información, haciendo especial hincapié en las fuentes de información usadas; 3 ) técnicas de análisis de la información, productos de inteligencia generados y difusión de inteligencia; y 4) generación de inteligencia y su contribución a la reforma de las titulaciones (tabla 2).

\subsection{Función y necesidades de información}

De nuestro estudio se desprende que la función de IC en la universidad está orientada a las necesidades tácticas, está descentralizada, y es reactiva.

Es táctica porque la observación del entorno y la aplicación de inteligencia están orientadas a temas relacionados con la actividad cotidiana de la universidad, sobre todo la necesaria para el proceso de adaptación al EEES. Esta orientación viene dada por las necesidades manifestadas por los entrevistados, causadas por tres factores: 1) el marco regulador nacional relacionado con el proceso de verificación, que obligaba a recopilar la opinión de los empleadores sobre la titulación diseñada y el perfil de los graduados, y justificar la idoneidad de la titulación a las necesidades del entorno;

\begin{tabular}{|c|c|c|}
\hline Necesidades & Fuentes & Inteligencia generada \\
\hline $\begin{array}{l}\text { - Justificación de la titulación de acuerdo con } \\
\text { las necesidades del entorno } \\
\text { - Conocer perfiles profesionales y } \\
\text { competencias requeridas por el entorno } \\
\text { - Funcionamiento de la titulación existente } \\
\text { en el mercado } \\
\text { - Objetivos estratégicos: } \\
\text { - ganar ventaja competitiva } \\
\text { - innovar: mejorar la titulación existente } \\
\text { - diseñar titulación nueva } \\
\text { - Conocer la competencia } \\
\text { - Detectar oportunidades de mercado }\end{array}$ & $\begin{array}{l}\text { - Consultas a agentes sociales } \\
\text { - Libro blanco } \\
\text { - Dossiers de prensa del Gabinete } \\
\text { - Informes de integración de los titulados al } \\
\text { mercado laboral* } \\
\text { - Informes de evolución de matriculados* } \\
\text { - Planes de estudio de otras universidades } \\
\text { - nacionales y extranjeras } \\
\text { - Informes de planes piloto } \\
\text { - Prospectivas socioeconómicas de la zona de } \\
\text { influencia de la titulación } \\
\text { * elaboración de la Unidad de Calidad, o la de Marketing }\end{array}$ & $\begin{array}{l}\text { - Portafolio de titulaciones de la } \\
\text { universidad } \\
\text { - Diseño de la oferta formativa: plan de } \\
\text { estudios, competencias y perfiles } \\
\text { - Memoria del grado: justificación y perfil } \\
\text { - Acciones a largo plazo: incorporación } \\
\text { de agentes sociales externos en la } \\
\text { actividad evaluadora de los centros }\end{array}$ \\
\hline
\end{tabular}

Tabla 2. Resumen del ciclo de inteligencia en las universidades 
2) los objetivos estratégicos de cada universidad, facultad, escuela o departamento, vinculados a la visión de proceso de adaptación: como una oportunidad de innovar para ganar ventaja competitiva y mejorar, o como un mero trámite administrativo, necesario para seguir impartiendo la titulación; y 3) la percepción que cada universidad o centro tenía de la competencia en su área de influencia, de manera que a mayor competencia mayor intensidad aplican en la búsqueda de información.

La responsabilidad de recoger información y analizarla recayó en diferentes unidades o departamentos. Hay predominio de las unidades de calidad como responsables de la gestión de información, tanto interna como externa, con predominio de la primera, y con definición de unos procedimientos establecidos. Otras unidades encargadas de gestionar información son el consejo social, la unidad de marketing, y en menor medida la biblioteca y el observatorio de empleo, incipiente en algunas universidades. A parte de estas estructuras, la función de inteligencia está distribuida entre personas de distintos departamentos. Pocas universidades han creado un grupo específico para buscar y analizar información para el proceso de adaptación. Esta escasa organización de la función ha originado cierta dispersión, que ha causado en algunos centros duplicidad de datos y mayor esfuerzo en la localización de información ya existente en la universidad.

La motivación de las prácticas de IC es reactiva porque son una respuesta a la incertidumbre del entorno, y a las amenazas detectadas del entorno, y por tanto, sin frecuencia fija de búsqueda de información. Pocas universidades han manifestado una orientación proactiva, con el objetivo de diseñar acciones estratégicas, y anticiparse a posibles amenazas.

\subsection{Recogida de información y fuentes empleadas}

La obtención de información se ha efectuado tanto por medios formales, como informales, y mediante sistemas de información manuales y tecnológicos.

Las fuentes utilizadas para obtener información han sido tanto las escritas como las orales, formales e informales, aunque con un papel destacado del capital social de las universidades, es decir la red de profesionales y empleadores con los que está vinculada la propia universidad, en su actividad habitual. En concreto, han sido consultados expertos del sector o sectores a los que se vincula la titulación, estudiantes, alumnos, profesores de otras universidades, y en menor medida estudiantes de secundaria. Las consultas se han hecho tanto de manera individualizada (presencialmente o por correo electrónico), como colectiva. Esta última fórmula ha permitido intercambiar más información, de cara a diseñar un grado adecuado a los perfiles profesionales que requiere el mercado de trabajo actual, tanto del sector privado como público.

Algunas universidades han buscado nuevos agentes sociales, además del capital social ya existente en la universidad, para que participasen en estructuras de trabajo específicas para la definición del grado. Este grupo de universidades han manifestado voluntad más innovadora, y han considerado el proceso de adaptación como una oportunidad.
Como fuentes escritas más utilizadas destacan el Libro blanco de la titulación, los informes de la unidad de calidad o equivalente $y$, en menor medida, estudios del departamento de marketing y del consejo social, dossiers de prensa, informes elaborados por el gabinete de la universidad, y los planes de estudio de otras universidades, tanto nacionales como internacionales. Pocas universidades han utilizado el servicio del gabinete de prensa para observar sistemáticamente el entorno sobre temas de interés. Algunas universidades utilizaron también productos informativos elaborados previamente, fruto del esfuerzo de planes piloto o procesos de autoevaluación anteriores.

En general, las universidades con voluntad innovadora han empleado más fuentes tanto escritas como humanas, con el objetivo de diferenciar sus grados de los de la competencia.

En cuanto al sistema de organización de la información recogida, hay tendencia a usar un repositorio o una intranet. No obstante, es incipiente la existencia de procedimientos de gestión de la información que aún están sin incluir en los sistemas de gestión de la calidad existentes en cada universidad. Además, es significativo el carácter individual del archivado de documentos y el intercambio de información informal mediante correos electrónicos.

\subsection{Análisis de información, productos de inteligen- cia y difusión}

En general, el análisis de información ha sido poco sistemático y las técnicas empleadas sencillas. Pocos entrevistados han manifestado que la información era analizada colectivamente en comisiones o consejos de gobiernos. Incluso hemos detectado falta de procedimientos sistemáticos para crear conocimiento e inteligencia y difundirla. Solo los departamentos que tenían como objetivo reformar sus titulaciones de manera innovadora han utilizado técnicas de análisis específicas como el DAFO y el benchmarking.

La elaboración sistemática de productos de inteligencia en las universidades aún es incipiente, a pesar de la existencia de unidades de información y la práctica generalizada de recogida de información, promovida, en parte, por los sistemas de calidad. En general, los productos de inteligencia elaborados por la misma universidad son informes sobre la actividad diaria: número de graduados, número de estudiantes matriculados y su evolución, grado de integración de los titulados en el mercado laboral, y, en menor medida, competencias requeridas a los titulados por el mercado de trabajo. Pocas universidades contrataron a empresas externas de estudios de mercado o prospectiva de evolución del entorno socio-económico.

\subsection{Generación de inteligencia y su contribución}

La generación de inteligencia y por tanto la contribución de ésta al proceso de adaptación al EEES, la constatamos en dos direcciones. Por un lado el diseño de la oferta formativa, en cuanto a decidir qué titulaciones se incluyen en el proceso de adaptación, cuáles se eliminan, y cuáles se reforman de manera profunda. Por otro, la elaboración de la memoria de cada grado entregada a la Agencia Nacional de Evaluación de la Calidad y Acreditación (Aneca) para ser verificada, 
que constituye un compendio de conocimiento para la acción surgido del análisis de la información. Los temas concretos son la justificación de la titulación a las necesidades del entorno, y el perfil de los graduados y sus competencias, adaptados a los requerimientos expresados por el mercado de trabajo, y mejorados de acuerdo con las opiniones recogidas en las consultas o en las fuentes de información revisadas.

Hemos percibido diferentes grados de inteligencia generada. Las universidades con un volumen consolidado de estudiantes y sin percepción de tener competencia han generado menos inteligencia en comparación con las que han percibido mayor presión del entorno (causada por tener competencia de otras universidades o por la disminución de matriculados).

Por último, señalamos la existencia de un grupo reducido de universidades que han aplicado inteligencia con orientación estratégica. Además de aplicar la inteligencia al diseño de acciones a corto plazo, y a la toma de decisiones relacionada con el proceso de adaptación, ese grupo ha diseñado acciones estratégicas a largo plazo. Un ejemplo es el proyecto de incluir a agentes sociales externos en procesos de evaluación del grado en vistas a la renovación de su acreditación.

\section{Discusión y conclusiones}

Los resultados obtenidos apuntan prácticas embrionarias en la definición y organización de la función y el ciclo de inteligencia en las universidades, influenciadas por el emergente valor dado a la gestión estratégica de la información, pero son predominantemente tácticas, descentralizadas, descoordinadas y reactivas.

Desde el punto de vista de la función, la orientación táctica de la inteligencia y su ubicación descentralizada confirman una tendencia detectada también por otros estudios, que apuntan tal descentralización como la fórmula idónea para la orientación táctica de la inteligencia (Saayman et al., 2008; Sawka, 2001). Sin embargo, a pesar de la bondad de esta fórmula, la falta de procedimientos de coordinación de esfuerzos causa dispersión de las herramientas de gestión y fragmentación/duplicidad de la información sobre el entorno, lo que disminuye la eficacia de las prácticas de IC. Esto concuerda así mismo con estudios previos llevados a cabo en otros sectores de actividad (Heppes; Du-Toit, 2009; Fahey, 2007).

El predominio de prácticas reactivas tácticas, sin periodicidad establecida, orientadas a reducir la incertidumbre, más que a detectar oportunidades, puede ser debido a la aún primitiva cultura en inteligencia competitiva.

A nivel organizativo contrasta la metódica recogida y análisis de información interna -contemplada en los sistemas de gestión de la calidad-con la irregular recogida de información del exterior, y la falta de sistemática en su análisis y explotación. Mientras es usual la existencia de una unidad de calidad con unos procedimientos bien definidos, pocas universidades tienen coordinada y procedimentada la función de inteligencia. Destaca el distinto nivel de madurez de las dos funciones.
La caracterización de las prácticas de IC que hemos presentado es indistinta para las universidades de titularidad pública y privada, históricas y de nueva creación, siendo las diferencias entre universidades motivadas por aspectos de otra índole, tanto internos como externos. Concretamente, entre las causas que han motivado a las universidades a incorporar o intensificar algunos instrumentos de gestión estratégica de la información están el incremento de la incertidumbre del entorno y la redefinición de los objetivos estratégicos -de entre los cuales destacan: innovación, surgimiento de un problema específico, percepción de competencia en el entorno, o necesidad de anticiparse a los problemas-. Por tanto, el ambiente de presión, de cambio e incertidumbre originado por el proceso de adaptación de las titulaciones al EEES ha impulsado las prácticas de inteligencia, coincidiendo con estudios previos de organizaciones, pertenecientes a otros ámbitos (El Mabrouki, 2007).

El contexto de urgencia y precipitación del proceso de adaptación de los grados al EEES no ha sido un factor inhibidor de las prácticas de IC, ya que éstas han quedado de manifiesto tanto en las titulaciones presentadas en primera convocatoria como en ulteriores. Sí puede haberlo sido, en cambio, la percepción del proceso de adaptación como un mero trámite administrativo.

Contrasta la metódica recogida y análisis de información interna -contemplada en los sistemas de gestión de la calidadcon la irregular recogida de información del exterior, y la falta de sistemática en su análisis y explotación

Para favorecer la maduración de la función de inteligencia en las universidades, éstas precisan incluir procedimientos transversales a toda la organización que engloben el ciclo de inteligencia. En concreto: cómo detectar las necesidades de información del entorno, cómo organizar ésta información, qué técnicas de análisis son adecuadas a los objetivos, y qué productos de inteligencia se deben generar para satisfacer los objetivos de la universidad y de los centros.

El modelo detectado en este estudio es un punto de partida para investigaciones posteriores, considerando dos líneas de trabajo:

- estudiar si estas prácticas llevadas a cabo para adaptar los planes de estudio son coyunturales, si han servido para implementar sistemas estructurales, o si están implementadas en otros ámbitos de gestión de las universidades;

- estudiar si la consolidación de las prácticas de IC y su mejor explotación constituyen una línea de trabajo sólida que pudiera facilitar la planificación y la toma de decisiones en distintos ámbitos de las universidades.

Un punto de partida es la vinculación de las prácticas de IC con los planes de calidad y los planes estratégicos ampliamente extendidos en las universidades.

La IC se perfila como una herramienta de gestión necesaria para que las universidades puedan cumplir el papel que 
tienen asignado en el desarrollo de la región donde están ubicadas, atendiendo a su misión docente, de investigación y de transferencia de conocimiento.

\section{Nota}

1. La construcción de sentido es el proceso por el cual las personas dan sentido a la experiencia. Si bien este proceso ha sido estudiado en otras disciplinas, bajo otros nombres, durante siglos, desde la década de 1970 el término "construcción de sentido" ha marcado principalmente tres áreas de investigación diferentes pero relacionadas: la interacción persona-ordenador, la ciencia de la información, y los estudios organizacionales (Karl Weick).

El concepto se ha utilizado para reunir ideas extraídas de la filosofía, la sociología y las ciencias cognitivas (especialmente la psicología social).

http://en.wikipedia.org/wiki/Sensemaking

\section{Agradecimientos}

Esta investigación ha sido financiada por el Ministerio de Educación de España, Estudios y análisis para la mejora del sistema universitario (EA-2008-0152).

\section{Bibliografía}

Bergeron, Pierrette; Hiller, Christine A. "Competitive intelligence". Annual review of information science and technology, 2002, v. 36, n. 1, pp. 353-390.

http://dx.doi.org/10.1002/aris.1440360109

Cartwright, Donna L.; Boughton, Paul D.; Miller, Stephen W. "Competitive intelligence systems: relationships to strategic orientation and perceived usefulness". Journal of managerial issues, 1995, v. 7, n. 4, pp. 420-434.

Cobarsí, Josep; Bernardo, Mercè; Coenders, Germà. "Campus information systems for students: classification in Spain". Campus-wide information systems, 2008, v. 25, n. 1, pp. 50-64.

Choo, Chun-Wei. Information management for the intelligent organization: the art of scanning the environment. Medford, New Jersey: Information Today, 2002.

Cronin, Blaise. "The intelligent campus: competitive intelligence and strategic planning". En $20^{\text {th }}$ Annual conf of the Indiana Association for Institutional Research (Inair), Indiana, Mars 2006.

Culver, Martha. "Using tactical intelligence to help inform strategy". Strategy \& leadership, 2006, v. 34, n. 6, pp. 17-23.

Dishman, Paul L.; Calof, Jonathan L. "Competitive intelligence: a multiphasic precedent to marketing strategy". European journal of marketing, 2008, v. 42, n. 7/8, pp. 766-785. http://dx.doi.org/10.1108/03090560810877141

El-Mabrouki-Nabil, Mohammed. "La practique de I'intelligence économique dans les grandes enterprises: voyage au coeur d'un système non univoque". En: XVlème Conf internationale de management stratégique, 2007.

Fahey, Liam. "Connecting strategy and competitive intelligence: refocusing intelligence to produce critical strategy inputs". Strategy \& leadership, 2007, v. 35, n. 1, pp. 4-12.

Fleisher, Craig S. "Using open source data in developing competitive and marketing intelligence". European journal of marketing, 2008, v. 42, n. 7/8, pp. 852-866. http://dx.doi.org/10.1108/03090560810877196

Fleisher, Craig S.; Bensoussan, Babette E. Business and competitive analysis: effective application of new and classic methods. Upper Saddle River, New Jersey: Pearson Education, Inc., 2007.

Hammond, Kevin L.; Harmon, Harry A.; Webster, Robert L. "University performance and strategic marketing: an extended study". Marketing intelligence \& planning, 2007, v. 25, n. 5, pp. 436-459.

Heppes, David; Du-Toit, Adeline. "Level of maturity of the competitive intelligence function: case study of a retail bank in South Africa". Aslib proceedings: new information perspectives, 2009, v. 61, n. 1, pp. 48-66.

http://dx.doi.org/10.1108\%2F00012530910932285

Larsen, Ingvild M.; Maassen, Peter; Stensaker, Bjørn. "Four basic dilemmas in university governance reform". Higher education management and policy, 2009, v. 21, n. 3, pp. 4158.

http://dx.doi.org/10.1787\%2Fhemp-21-5ksdxgpdnds1

Marin, Jane; Poulter, Alan. "Dissemination of competitive intelligence". Journal of information science, 2004, v. 30, n. 2, pp. 165-180.

http://dx.doi.org/10.1177\%2F0165551504042806

Michaeli, Rainer. Competitive intelligence: strategische Wettbewerbsvorteile erzielen durch systematische Konkurrenz-, Markt-und Technologieanalysen. Springer, Berlin, Heidelberg 2006. ISBN: 9783540030812.

Ortoll, Eva; López-Borrull, Alexandre; Canals, Agustí; Garcia-Alsina, Montserrat; Cobarsí-Morales, Josep. "El papel del capital social en la inteligencia competitiva". Revista general de información y documentación, 2010, v. 20, n. 1, pp. 313-323.

Ortoll, Eva; López-Borrull, Alexandre; Cobarsí-Morales, Josep; Garcia-Alsina, Montserrat; Canals, Agustí. "El capital social com a font d'intel-ligència competitiva a les universitats". UOC Papers, 2008, n. 7.

http://www.uoc.edu/uocpapers/7/dt/cat/ortoll_lopez_cobarsi_garcia_canals.pdf

Ortoll, Eva; Garcia-Alsina, Montserrat. La intel-ligència competitiva. Barcelona: Editorial UOC, 2008. Col. Vull saber.

Saayman, Andrea; Pienaar, Jaco; De-Pelsmacker, Patrick; Viviers, Wilma; Cuyvers, Ludo; Muller, Marie-Luce; Jegers, Marc. "Competitive intelligence: construct exploration, validation and equivalence". Aslib proceedings: new information perspectives, 2008 , v. 60, n. 4, pp. 383-411.

http://dx.doi.org/10.1108\%2F00012530810888006

Sawka, Kenneth A. "Deciding where to locate the intelligence unit". En Miller, J. P. Millennium intelligence: understanding and conducting competitive intelligence in the digital age. Medford, New York: CyberAge books, 2001. ISBN: 0910965-28-5 